\title{
S Research Square
Information Transmission among Multiple \\ Investors: A Micro Perspective Revealed by Motifs
}

Jie Li

Nanjing Audit University

Yongjie Zhang

Tianjin University

Lidan Wang ( $\nabla$ wanglidan@nau.edu.cn )

Nanjing Audit University https://orcid.org/0000-0002-7261-0786

\section{Research Article}

Keywords: shareholder co-holding network, motif identification, motif evolution, information transmission

Posted Date: July 15th, 2021

DOI: https://doi.org/10.21203/rs.3.rs-699505/v1

License: (1) This work is licensed under a Creative Commons Attribution 4.0 International License.

Read Full License

Version of Record: A version of this preprint was published at Nonlinear Dynamics on February 24th, 2022. See the published version at https://doi.org/10.1007/s11071-022-07307-8. 


\title{
Information transmission among multiple investors: a micro perspective
} revealed by motifs

\author{
Jie Li $^{\text {a }}$, Yongjie Zhang ${ }^{\mathrm{b}}$, Lidan Wang ${ }^{\mathrm{c}, 1}$ \\ ${ }^{a}$ School of Finance, Nanjing Audit University, Nanjing, Jiangsu 211815, China \\ ${ }^{\mathrm{b}}$ College of Management and Economics, Tianjin University, Tianjin 300072, China \\ c School of Government Audit, Nanjing Audit University, Nanjing, Jiangsu 211815, China
}

\begin{abstract}
We build the shareholder co-holding network (SCN) based on common shareholding data from 2007 to 2017. Considering the node attributes and the link weights, we reveal the basic structure and dynamic evolution of the SCN from two aspects: motif identification and motif evolution. Research on motif identification shows that although closed motifs have a low proportion, they are statistically significant. Further, research on the motif evolution shows that all motif structures have a higher tendency to disappear. The motifs containing financial investment company shareholders tend to disappear, while the motifs containing general corporate shareholders tend to remain unchanged during the evolution process. In short, we have developed the local motif structure of the SCN, which helps understand how information is transmitted among multiple investors.
\end{abstract}

Keywords: shareholder co-holding network; motif identification; motif evolution; information transmission

\section{Introduction}

The studies of small-world networks and scale-free networks opened the prelude to the study of complex networks[1,2]. The object of complex network research is nodes and the relationship between nodes [3]. The social network is an essential branch of complex network research, and the research objects are social members and their relationships [4]. Research on social networks has developed rapidly in recent years, mainly due to the apparent relationship between nodes. Specifically, nodes are participants in social networks, and the relationship between nodes is a specific connection between participants, for example, alumni relationship [5], professional relationship [6], geographic relationship [7], and communication relationship [8], etc.

\footnotetext{
${ }^{1}$ Corresponding author at: School of Government Audit, Nanjing Audit University, Nanjing, Jiangsu 211815, China.

E-mail address: wanglidan@nau.edu.cn
} 
Recently, scholars have begun to pay attention to social networks among shareholders. Since shareholders' social or geographic relationship is relatively difficult to obtain, a large number of studies have established the relationship between shareholders by extracting the mutual shareholding relationship between shareholders [9-11]. The SCN is a network among shareholders established by the behavior of jointly holding stocks. Existing academic research confirms that SCN is a private information network between shareholders from investor information interaction [12-15]. Then, scholars explore the impact of information interaction in SCN on market risk [9], investor decision making [10], and company performance [11] from a global perspective. In addition to these valuable works with a global perspective, more details need to be explored through a micro perspective. For example, are there specific microstructures that play an essential role in the transmission of information? How does the microstructure in the network evolve in time series?

Milo et al. (2002) introduced the concept of "network motif" in the study of Escherichia coli networks and defined it as the basic pattern that repeatedly appears in the networks [16]. The frequency of motif in real networks is significantly higher than that in random networks. They pointed out that the motif, as the basic building block of the network, plays an important role in information transmission. For example, in biological networks, the motif is usually a functional structure reserved and preferred during evolution [16,17]. Existing studies have explored the motif structures of complex networks in different fields, including biological systems networks [18], social networks [19], electronic communication networks [20], stock time series networks [21], and listed companies networks [22]. However, there is relatively little research on the mining of motif structure in the SCN. Guan et al. (2017) explored the motif structure of the SCN of listed companies in the energy industry based on annual shareholding data [23]. This paper will mine the motif structure of the SCN using higher frequency and more comprehensive sample data.

Due to differences in shareholders' investment objectives [24], risk preferences [25], capital levels [26], and company decision-making participation levels [27], the investment behaviors of different types of shareholders often show significant differences. Therefore, it is necessary to distinguish the types of shareholders in the study of a co-holding relationship. Besides, due to the different speeds of information transmission between different shareholders [24], it is necessary to divide the strength of co-holding between shareholders in the study coholding relationship. In this article, we divide the nodes into five attributes: financial investment companies (FIC), state-owned companies (SOC), general companies (GC), 
qualified foreign institutional investors (QFII), and individual investors (II). Also, we divide the links into two types: strong ones and weak ones. Considering node attributes and link types, we reveal the basic structure and dynamic evolution of SCN from two aspects: motif identification and motif evolution.

Based on motif identification research, we find that the proportion of closed motif in the SCN is significantly higher than that in the random network, although its proportion is relatively low. Further considering the node attributes, the motifs with all three nodes being FIC shareholders have the highest proportion. We distinguish three evolution states of motif invariant, motif vanishing, and motif transforming based on motif evolution research. All motif structures are found to have a higher tendency to vanish. The longer the period, the greater the possibility of disappearance. Further, considering the node attributes, motifs containing FIC nodes have a higher tendency to disappear, while motifs containing GC nodes tend to keep the structure unchanged.

The innovation of this paper is to explore the basic structure and dynamic evolution of the SCN. Guan et al. (2017) examined the motif structure of the SCN based on the annual shareholding data of listed companies in the energy industry [23]. Due to low-frequency data sampling (only yearly) and limited research objects (energy industry only), their results are not representative of the overall market. In contrast, we use higher frequency sampling data (quarterly) and a more comprehensive stock sample (CSI 300 Index). More importantly, we consider the link strength, node attributes, and different time scales to explore the motif structure and dynamic evolution. More detailed and market representative conclusions are obtained.

The remainder of this paper is organized as follows. Section 2 describes data and modeling. Section 3 reports results and analysis classified into two parts: motif identification and motif evolution. Section 4 concludes the paper.

\section{Data and modeling}

\subsection{Data}

The data of the top ten shareholders of tradable stocks comes from the RESSET database. The time is from the first quarter of 2007 to the fourth quarter of $2017^{2}$. The samples are the

${ }^{2}$ As of the end of 2006, 1301 companies had completed the split share reform, accounting for $97 \%$ of the total number of listed companies. In order to exclude the influence of shareholder structure and equity nature on the results, the starting point of the data in this paper is the first quarter of 2007. 
constituent stocks of the CSI 300 index and are updated every quarter ${ }^{3}$. Since the same shareholders' names may differ in different company documents, we manually unify them for consistency. As for the various subsidiaries of the same parent company, since they are acting in concert, we consider them to be the same shareholder. Besides, index funds and indexenhanced funds only track an index for passive holding and often have no information interaction with other shareholders. Also, controlling shareholders hold shares to gain control of the company, which is different from ordinary shareholders' purpose of making profits based on private information. We delete these two types of shareholders to exclude their actions from interfering with the results.

According to Li et al. (2009), we divide shareholders into five types: FIC, SOC, GC, QFII, and II [28]. The classification standard for shareholder categories comes from the RESSET database.

\subsection{The SCN modeling}

$\mathrm{SCN}$ is used to describe the relationship between investors. According to the network construction method in Newman (2001), shareholders are taken as the node, whether the shares are jointly held is the edge, and the number of shares together held is the edge weight [29]. In other words, SCN is a weighted but undirected network used to describe collective holding behavior among investors. Using $\mathrm{H}=\left\{\mathrm{H}_{1}, \mathrm{H}_{2}, \cdots, \mathrm{H}_{\mathrm{n}}\right\}$ to represent shareholders, matrix $\mathrm{A}$ describes the SCN as follows:

$$
\mathrm{A}= \begin{cases}\mathrm{a}_{\mathrm{ij}}=w_{i j} & \mathrm{H}_{\mathrm{i}} \text { and } \mathrm{H}_{\mathrm{j}} \text { jointly hold } \mathrm{w}_{\mathrm{ij}} \text { listed companies } \\ \mathrm{a}_{\mathrm{ij}}=0 & \mathrm{H}_{\mathrm{i}} \text { and } \mathrm{H}_{\mathrm{j}} \text { jointly hold no listed companies }\end{cases}
$$

where $w_{i j}$ is the number of listed companies that shareholders $H_{i}$ and $H_{j}$ jointly hold.

\subsection{Motif modeling}

\subsubsection{Motif in co-shareholding network}

Motifs are the basic units of a complex network system, so their repeated appearance constitutes the entire network. The motif based on three nodes is the most common subgraph form in the network [16]. In undirected and unweighted networks, there are only two forms of 3-motifs, including motif 1 and motif 4 of Figure 1. We divide the links between shareholders in the SCN into strong and weak links. Shareholders jointly hold one stock as weak links and share multiple stocks as strong links. In the SCN, strong and weak links have profound

3 The CSI 300 index consists of 300 of the largest and most liquid A-shares. The index is designed to reflect the overall performance of China's A-share market. 
economic meanings, representing information transmission speed among shareholders. When considering the link strength, the 3-motifs have seven forms, as shown in Figure 1.

\section{[Insert Figure 1 here]}

Motifs 1, 2, and 3 have two nodes that are not directly connected but indirectly related through intermediate nodes to form a chain structure, called the chain motifs. In contrast, motifs 4, 5, 6, and 7 are connected to form a closed structure, called the closed motifs. As the shape of the motif changes from chain to closed and the weight of the link changes from weak to strong, the co-holding behavior among shareholders gradually increases, which leads to the speed of information transmission of the motif increasing progressively.

We also consider the node attributes in the SCN and divide shareholders into five types: FIC, SOC, GC, QFII, and II. Therefore, each node in the 3-motifs has five different attributes. Further, different node locations also lead to different forms of the motif. Take motif 2 as an example. Due to the chain structure and different weights of links, motif 2 will have up to 125 $(5 \times 5 \times 5)$ shapes when considering node attributes. Figure 2 shows the schematic figure of motif identification when considering both link weights and node attributes.

\section{[Insert Figure 2 here]}

Fanmod Software is used for motif identification in this paper. First, perform the motif identification process shown in Figure 2 on the real network to calculate the proportion of various motifs; Secondly, perform 1000 simulations on random networks of the same scale to calculate the proportion of various motifs; Finally, calculate the motif statistical significance according to the proportion of various phantoms in the real network and the random network.

\subsubsection{Motif with statistical significance}

Motifs are the basic units that make up a network. Three common types of indicators measure the importance of motifs in networks, including the frequency of motifs, the p-value of motifs, and the Z-score of motifs.

(1) The frequency of the motif

For a motif $\mathrm{M}$ with $\mathrm{n}$ nodes, the number of occurrences in the real network is $\mathrm{n}(\mathrm{M})$, and the total number of occurrences of all subgraphs with $\mathrm{n}$ nodes is $\mathrm{N}$, then the frequency of the motif $\mathrm{M}$ is as follows.

$$
f(M)=\frac{n(M)}{N}
$$

(2) The p-value of the motif 
The $\mathrm{p}$-value of the motif refers to the number of times that a specific motif appears more frequently in the random network than in the real network divided by the total number of random networks ${ }^{4}$. Therefore, the p-value of the motif is between 0 and 1 . The smaller the $\mathrm{p}$ value, the more significant the motif, and the more critical it is in the real network.

(3) The Z-score of the motif

The Z-score of the motif is an index proposed by Milo et al. (2002) to measure the significance of the motif [16]. For motif M, the number of times it appears in the real network is $N_{\text {real }_{M}}$, the number of times it appears in the random network is $N_{\text {rand }_{M}}$, and the standard deviation is $\sigma_{\text {rand }_{M}}$. The average value of $N_{\text {rand }_{M}}$ is $\left\langle N_{\text {rand }}\right\rangle$. The Z-score of motif M in the real network is as follows.

$$
Z_{M}=\frac{N_{\text {real }_{M}}-\left\langle N_{\text {rand }_{M}}\right\rangle}{\sigma_{\text {rand }_{M}}}
$$

The construction logic of the Z-score and p-value is similar. They both compare the frequency of the motif in the real and random networks and use the difference to measure the importance of the motif.

\section{Results and analysis}

\subsection{Summary statistics}

We construct the SCN based on the shareholding data of the constituent stocks of the CSI 300 Index from 2007 to 2017. The descriptive statistics are conducted from the three perspectives of nodes, links, and the overall network.

Panel A of Table 1 shows the summary statistics of different types of shareholder holdings. FIC shareholders are the most frequent, accounting for about $62 \%$, while SOE shareholders are the least frequent. QFII shareholders hold the largest number of shares on average, while SOE shareholders hold the highest average market capitalization of their shares. Panel B shows the summary statistics of strong links. FIC shareholders have held multiple stocks together, among which FIC-FIC type of links accounted for $68.97 \%$ on average, indicating that there is sufficient information transmission between shareholders of the same kind. Panel C shows the summary statistics of weak links. The proportion of links containing FIC shareholders is the highest, which is related to the relatively high proportion of FIC nodes in the SCN. Similar to Panel B, the FIC-FIC type of links have the highest proportion. Panel D shows descriptive statistics of network topology characteristics.

\footnotetext{
4 The number of random network in this paper is 1000 .
} 


\section{[Insert Table 1 here]}

\subsection{Motif identification}

\subsubsection{Consider the link weight}

The Constitution of motifs helps to understand local information transmission among shareholders. In this part, without the consideration of node attributes, we explore seven weighted 3-motifs in the SCN from 2007 to 2017 . We calculated the frequency, p-value, and Z-score of each motif, respectively. Table 2 provides the results.

First, from the time series, motif 1 and motif 2 remain relatively stable, while the frequency of motif 5, motif 6 and motif 7 shows a gradual decrease. Second, the frequency of the chain motifs in the SCN is higher than that of the closed motifs. Moreover, as the number of strong links increases, the frequency of the motif decreases. Finally, the four types of closed motifs show statistical significance, while the chain motifs do not show significance, indicating that the closed motifs play an essential role in information transmission.

\section{[Insert Table 2 here]}

\subsubsection{Consider the node attribute}

Next, considering the node attributes, we further explore the components of the significant motifs (closed motifs) in the SCN. Table 3 lists the top five closed motif types with the highest frequency of occurrence in all quarters. The results show that the motifs of three nodes with the FIC attribute have the highest proportion. Other motifs with a high proportion also contain two FIC nodes. In short, FIC shareholders are an important component of the closed motif. Also, the proportion of closed motifs in 2013-2017 is lower than that of the other two periods, which may be related to the stock market crash in 2016. A large number of shareholders sold their shares, causing the motif to disappear.

\section{[Insert Table 3 here]}

\subsection{Motif evolution}

The stock market exhibits evident volatility, and the direct cause is investors' trading behavior, which is manifested in the generation and disappearance of motifs on a microscopic level. To deeply understand the volatility of the stock market, we explore the potential volatility laws from the dynamic evolution of motifs. The motif evolution is divided into the following three steps: First, calculate the shareholder intersection of the previous and subsequent periods. Secondly, excavate the local structure of motif 1-7 in the SCN formed by shareholder intersection in the previous period. Finally, mine the corresponding evolved motif structure in the later period. We select time scales of short period (quarterly) and long period (annual) to 
explore the characteristics of motif evolution in this part.

\subsubsection{Motif evolution in the short term}

The motif in the later period corresponds to three evolution states: invariant motif, vanishing motif, and transforming motif. Specifically, the invariant motif is one that keeps the same structure in two periods. The vanishing motif refers to at least one isolated node in the motif due to the edge fracture. The transforming motif means that the motif structure of the two periods is different due to the edge fracture or reconnection.

Figure 3 shows the results of the motif evolution on the quarterly time scale. The first column corresponds to the 3-node motif with at least one isolated node, representing the vanishing motif. The diagonal line is the same motif structure in the front and later periods, representing the invariant motif. The other figure positions are different motif structures in the early and later periods, representing the transforming motif.

\section{[Insert Figure 3 here]}

First, as shown in the first column, all motif structures have a higher tendency to disappear. As the link weight changes from weak to strong, and the structure changes from chain to closed, the motif's tendency to disappear gradually decreases. Second, as shown from the diagonal, all motif structures have a relatively high propensity to remain unchanged, especially motif 4 . Third, in the transforming motif, the transforming probability from a motif with high information transmission speed to a motif with low information transmission speed is higher. Still, the possibility of the reverse is relatively lower. In short, edges tend to break rather than reconnect. Such an evolution result is in line with the principle of entropy increase in physics. That is, the system always tends to transform from an ordered state to a disordered state.

Next, considering the node attributes, we count the composition of each motif evolution state. The result is shown in Figure 4. To ensure the accuracy of the results, we delete the motif types whose total number is less than 100. Among the invariant motifs, the proportion of yellow and green nodes is relatively high, indicating that the holdings of GC and II are stable and tend to remain unchanged during the motif evolution. Especially for motif 4, the probability that the motif with all three nodes as II remains unchanged during the quarterly evolution is 0.98 . Further, among the vanishing motifs, the motifs containing red nodes account for a relatively high proportion, indicating that FIC has active market transactions and is likely to disappear during the motif evolution. Finally, among the transforming motifs, the holdings of FIC and QFII are unstable, so motifs containing these two types of nodes are easily transformed into other varieties in the evolution process. 


\section{[Insert Figure 4 here]}

In short, the above analysis shows that during the quarterly evolution of motifs, motifs containing GC and II are stable, and motifs containing FIC tend to transform or even disappear. In contrast, motifs containing QFII tend to transform into other types.

\subsubsection{Motif evolution in the long term}

Next, we will explore the motif evolution from the long-period time scale (annual). The calculation steps are the same as those in the short period, except that the time window changes from quarter to year. The result is shown in Figure 5.

It can be seen from the first column of Figure 5 that all the motifs have a higher tendency to disappear. Compared with the motif evolution results in a short period, the probability of the disappearing motif in a long period is more outstanding. This result shows that investors' shareholding behavior is more unstable in a long period. Further, it can be seen from the diagonal that all the motifs have a relatively high tendency to remain unchanged. Still, the probability is reduced compared with the situation in a short period. Third, the transforming probability from a motif with high information transmission speed to a motif with low information transmission speed is higher in transforming motif. This result is the same as the conclusion of the short-period motif evolution. That is, the system always tends to evolve to a state of increasing entropy.

\section{[Insert Figure 5 here]}

Next, considering the node attributes, we count the composition of each motif evolution state. The result is shown in Figure 6. The conclusion of the motif evolution in the long period is the same as the conclusion in the short period. However, the probability of the motif evolution remaining unchanged in the long period is less than the probability in the short period. In contrast, the probability of the motif disappearing and transforming in the long period is greater than the probability in the short period. This conclusion conforms to the law of entropy increase. The longer the time, the higher the system chaos, and the greater the entropy.

\section{[Insert Figure 6 here]}

\section{Conclusion}

Based on the shareholding data from 2007 to 2017, we mine the local information of the SCN from the micro perspective. Specifically, we reveal the basic structure and dynamic evolution of SCN from two aspects: motif identification and motif evolution.

Considering the link weight, we find that although the proportion of closed motifs in SCN is low, it is statistically significant. Subsequently, considering the node attributes, we explore 
the composition of the closed motifs. The results show that the three nodes are all FIC shareholders with the highest proportion.

Based on motif evolution research, we select the time scales of the short period and the long period to explore motif evolution characteristics. At the same time, we distinguish three evolution states: invariant motif, vanishing motif, and transforming motif. First, all motif structures have a higher tendency to disappear. Moreover, the longer the time scale, the greater the possibility of disappearance. Besides, among the vanishing motifs, motifs containing FIC nodes account for a relatively high proportion. On the contrary, motifs containing GC nodes tend to keep their structure unchanged during the evolution process.

In conclusion, we have developed a micro perspective based on SCN research, which helps understand how information is transmitted in the network.

Acknowledgments: This article is funded by the National Natural Science Foundation of China (U1811462, 71771170, 71790594), Youth Foundation for Humanities and Social Sciences Research of the Ministry of Education (20YJC790062) and the Applied Economics of Nanjing Audit University of the Priority Academic Program Development Phase III of Jiangsu Higher Education Institutions (Office of Jiangsu Provincial People's Government, No. [2018]87).

\section{Declarations:}

\section{Ethics declarations}

The authors declare that the research was conducted in the absence of any commercial or financial relationships that could be constructed as a potential conflict of interest. The authors have no affiliation with any organization with a direct or indirect financial interest in the subject matter discussed in the manuscript.

\section{Availability of data and material}

The authors confirm that data will be made available on reasonable request.

\section{References}

[1] D. J. Watts, S. H. Strogatz, Collective dynamics of 'small-world' networks. Nature, 1998, 393(6684): 440-442.

[2] A. L. Barabasi, R. Albert, Emergence of scaling in random networks. Science, 1999, 
286(5439): 509-512.

[3] Z. Wang, L. Dueñas-Osorio, J. Padgett, A new mutually reinforcing network node and link ranking algorithm. Scientific Reports, 2015, 5, Article ID: 15141.

[4] B. Huberman, D. Romero, F. Wu, Social networks that matter: Twitter under the microscope. First Monday, 2009, 14(1).

[5] B. Schmidt, Costs and benefits of friendly boards during mergers and acquisitions. Journal of Financial Economics, 2015, 117(2): 424-447.

[6] K. Shue, Executive networks and firm policies: Evidence from the random assignment of MBA peers. Review of Financial Studies, 2013, 26(6): 1401-1442.

[7] H. Hong, J. D. Kubik, J. C. Stein, Thy neighbor's portfolio: Word-of-Mouth effects in the holdings and trades of money managers. The Journal of Finance, 2005, 60(6): 2801-2824.

[8] L. Kovanen, K .Kaski, J. Kertesz, J. Saramaki, Temporal motifs reveal homophily, genderspecific patterns, and group talk in call sequences. Proceedings of the National Academy of Sciences, 2013, 110(45): 18070-18075.

[9] A. Pareek, Information networks: Implications for mutual fund trading behavior and stock returns. Working paper, 2012.

[10]H. N. Ozsoylev, J. Walden, M. D. Yavuz, R. Bildik, Investor networks in the stock market. Review of Financial Studies, 2014, 27(5): 1323-1366.

[11]L. Qi, L. Wang, W. Li, Do mutual fund networks affect corporate social responsibility? Evidence from China. Corporate Social Responsibility and Environment Management, 2020, 27(2): 1040-1050.

[12]V. K. Pool, N. Stoffman, S. E. Yonker, The People in your neighborhood: Social interactions and mutual fund portfolios. The Journal of Finance, 2015, 70(6): 2679-2732.

[13]J. Engelberg, P. Gao, C. A. Parsons, Friends with money. Journal of Financial Economics, 2013, 103(1): 169-188.

[14]C. Fracassi, Corporate finance policies and social networks. Management Science, 2017, 63(8): 2420-2438.

[15]H. Hong, J. Xu, Inferring latent social networks from stock holdings. Journal of Financial Economics, 2019, 131(2): 323-344.

[16] R. Milo, S. Shen-Orr, S. Itzkovitz, N. Kashtan, D. Chklovskii, U. Alon, Network motifs: simple building blocks of complex networks. Science, 2002, 298(5594): 824-827.

[17]T. I. Lee, N. J. Rinaldi, F. Robert, et al. Transcriptional regulatory networks in Saccharomyces Cerevisiae. Science, 2002, 298(5594): 799-804.

[18]W. Niemyska, P. Dabrowski-Tumanski, M. Kadlof, E. Haglund, P. Sułkowski, J. I. Sulkowska, Complex Lasso: new entangled motifs in proteins. Scientific Reports, 2016, 110(3): 379-388. 
[19] W. J. Xie, M. X. Li, Z. Q. Jiang, W. X. Zhou, Triadic motifs in the dependence networks of virtual societies. Scientific Reports, 2015, 4(1), Article ID: 5244.

[20]L. Kovanen, K. Kaski, J. Kertesz, J. Saramaki, Temporal motifs reveal homophily, genderspecific patterns, and group talk in call sequences. Proceedings of the National Academy of Sciences, 2013, 110(45): 18070-18075.

[21]C. Curme, M. Tumminello, R. N. Mantegna, H. E. Stanley, D. Y. Kenett, Emergence of statistically validated financial intraday lead-lag relationships. Quantitative Finance, 2015, 15(8): 1375-1386.

[22]F. W. Takes, W. A. Kosters, B. Witte, E. M. Heemskerk, Multiplex network motifs as building blocks of corporate networks. Applied Network Science, 2018, 3(1), Article ID: 39.

[23]Q. Guan, H. An, N. Liu, F. An, M. Jiang, Information connections among multiple investors: Evolutionary local patterns revealed by motifs. Scientific Reports, 2017, 7(1), Article ID: 14034.

[24]Z. Ivkovich, S. J. Weisbenner, Information diffusion effects in individual investors' common stock purchases covet thy neighbors' investment choices. Review of Financial Studies, 2007, 20(4): 1327-1357.

[25]J. Li, D. Ren, X. Feng, Y. Zhang, Network of listed companies based on common shareholders and the prediction of market volatility. Physica A: Statistical Mechanics and its Applications, 2016, 449: 224-232.

[26]B. Jaggi, W. Li, S. Wang, Individual and institutional investors' response to earnings reported by conservative and non-conservative firms: Evidence from Chinese financial markets. Journal of International Financial Management \& Accounting, 2016, 27: 158-207.

[27]A. Ekholm, How do different types of investors react to new earnings information? Journal of Business Finance \& Accounting, 2006, 33(1-2): 127-144.

[28]J. Li, Y. Zhang, X. Feng, Y. An, Which kind of investor causes comovement? Journal of International Financial Markets, Institutions and Money, 2019, 61: 1-15.

[29]M. E. Newman, Scientific collaboration networks. I. Network construction and fundamental results. Physical Review E, 2001, 64(1), Article ID: 016131. 
Table 1. Summary statistics

Panel A shows summary statistics of different types of shareholder holdings. FH represents the frequency of shareholders, PS represents the proportion of shareholders, NH represents the number of shareholders, SHS represents the number of stocks held by shareholders, TMC represents the total market capitalization of stocks held by shareholders, and AMC represents the average market capitalization of stocks held by shareholders. Panel B shows the summary statistics of the top five strong links. FIC-FIC represents a strong link between two nodes whose attributes are FIC. Other links have similar meanings. Panel C shows the summary statistics of the top five weak links. Panel D shows summary statistics of network topology characteristics, including the number of edges (NE), number of nodes (NN), average weighted degree (AWD), average path length (APL), network diameter (NDia), clustering coefficient (CC), and network density (NDen). These characteristics are calculated following Guan et al. (2017). 


\begin{tabular}{ccccccc}
\hline \multicolumn{7}{c}{ Panel A: Summary statistics of shareholdings by different types of shareholders } \\
\hline Type & FH & PH & NH & SHS & TMC & AMC \\
\hline FIC & 1686.99 & 0.62 & 738.94 & 2.30 & $3.07 \mathrm{E}+12$ & $1.81 \mathrm{E}+09$ \\
GC & 406.87 & 0.15 & 346.54 & 1.17 & $1.64 \mathrm{E}+12$ & $4.00 \mathrm{E}+09$ \\
II & 348.21 & 0.13 & 340.41 & 1.02 & $2.42 \mathrm{E}+11$ & $6.99 \mathrm{E}+08$ \\
QFII & 245.50 & 0.09 & 66.02 & 3.76 & $3.78 \mathrm{E}+12$ & $1.53 \mathrm{E}+10$ \\
SOC & 31.61 & 0.01 & 20.11 & 1.22 & $1.12 \mathrm{E}+12$ & $7.83 \mathrm{E}+10$
\end{tabular}

Panel B: Summary statistics of the proportion of strong links

\begin{tabular}{ccccccc}
\hline & Mean & SD & $25 \%$ & $50 \%$ & $75 \%$ & Obs. \\
\hline FIC-FIC & 36.92 & 8.26 & 28.80 & 38.08 & 44.21 & 44 \\
FIC-GC & 13.29 & 3.57 & 10.54 & 13.21 & 15.66 & 44 \\
FIC-II & 10.32 & 3.95 & 6.98 & 8.29 & 13.90 & 44 \\
FIC-QFII & 8.01 & 1.05 & 7.51 & 7.60 & 8.33 & 44 \\
FIC-SOC & 8.00 & 3.73 & 5.81 & 7.81 & 11.62 & 44
\end{tabular}

Panel C: Summary statistics of the proportion of weak links

\begin{tabular}{ccccccc}
\hline & Mean & SD & $25 \%$ & $50 \%$ & $75 \%$ & Obs. \\
\hline FIC-FIC & 68.97 & 15.07 & 54.23 & 69.31 & 81.90 & 44 \\
FIC-QFII & 10.60 & 5.32 & 8.39 & 8.75 & 13.53 & 44 \\
FIC-SOC & 7.10 & 5.17 & 3.36 & 5.78 & 10.38 & 44 \\
FIC-GC & 4.84 & 2.90 & 2.96 & 4.88 & 5.75 & 44 \\
QFII-QFII & 2.61 & 2.00 & 1.35 & 1.73 & 3.26 & 44
\end{tabular}

Panel D: Summary statistics of network topology characteristics

\begin{tabular}{ccccccc}
\hline & Mean & SD & $25 \%$ & $50 \%$ & $75 \%$ & Obs. \\
\hline NE & 8234.98 & 574.46 & 7815.63 & 8258.75 & 8756.88 & 44 \\
NN & 1237.30 & 170.93 & 1086.75 & 1186.00 & 1360.50 & 44 \\
AWD & 13.48 & 1.25 & 12.68 & 13.20 & 14.04 & 44 \\
APL & 2.85 & 0.16 & 2.72 & 2.83 & 2.98 & 44 \\
NDia & 8.61 & 0.75 & 8.25 & 8.50 & 9.13 & 44 \\
CC & 0.22 & 0.07 & 0.19 & 0.23 & 0.27 & 44 \\
NDen $(\%)$ & 0.56 & 0.13 & 0.48 & 0.54 & 0.66 & 44 \\
\hline
\end{tabular}


Table 2. Significance of motif occurrences

Table 2 shows the significance of motif occurrences. Freq represents the frequency of the motif, and Z-score represents the $\mathrm{Z}$ score of the motif. The proportion of motifs in each year is the average of the four quarters, and the last row is the average of all quarters. *** indicates the significance level of $1 \%$.

\begin{tabular}{|c|c|c|c|c|c|c|c|c|c|c|c|c|c|c|}
\hline & Freq(\%) & Z-score & Freq $(\%)$ & Z-score & Freq $(\%)$ & Z-score & Freq(\%) & Z-score & Freq(\%) & Z-score & $\operatorname{Freq}(\%)$ & Z-score & Freq $(\%)$ & Z-score \\
\hline 2007 & 62.69 & -134.55 & 25.29 & -29.69 & 2.29 & -28.94 & $4.34 * * *$ & 138.79 & $3.27 * * *$ & 85.08 & $1.56^{* * *}$ & 73.13 & $0.57 * * *$ & 22.40 \\
\hline 2008 & 66.73 & -187.35 & 23.95 & -31.80 & 1.92 & -31.37 & $3.62 * * *$ & 172.71 & $2.44 * * *$ & 95.82 & $0.99 * * *$ & 68.05 & $0.34 * * *$ & 23.99 \\
\hline 2009 & 61.55 & -165.76 & 27.13 & -15.32 & 2.87 & -27.55 & $3.87 * * *$ & 144.28 & $2.83^{* * *}$ & 76.46 & $1.31^{* * *}$ & 70.59 & $0.44 * * *$ & 22.25 \\
\hline 2010 & 63.58 & -257.27 & 26.57 & -12.31 & 3.10 & -25.63 & $3.44 * * *$ & 192.98 & $2.18 * * *$ & 80.92 & $0.87 * * *$ & 62.05 & $0.27 * * *$ & 25.63 \\
\hline 2011 & 63.73 & -294.81 & 26.25 & -6.41 & 3.34 & -26.30 & $3.86^{* * * *}$ & 214.66 & $1.86^{* * * *}$ & 81.66 & $0.74 * * *$ & 60.57 & $0.22 * * *$ & 25.99 \\
\hline 2012 & 62.92 & -249.12 & 26.66 & -5.71 & 3.33 & -35.49 & $4.07 * * *$ & 212.72 & $1.97 * * *$ & 80.33 & $0.79 * * *$ & 70.82 & $0.26 * * *$ & 33.77 \\
\hline 2013 & 67.90 & -266.91 & 23.04 & -10.76 & 2.16 & -53.25 & $4.37 * * *$ & 289.66 & $1.78^{* * * *}$ & 83.20 & $0.58^{* * *}$ & 81.24 & $0.17 * * *$ & 50.94 \\
\hline 2014 & 70.53 & -378.90 & 19.99 & 3.62 & 1.84 & -55.66 & $5.47 * * *$ & 432.68 & $1.58^{* * *}$ & 95.61 & 0.4 & 90.95 & 0.11 & 62.70 \\
\hline 2015 & 66.75 & -219.27 & 25.12 & -27.23 & 2.38 & -30.64 & $3.80^{* * *}$ & 115.20 & $1.38^{* * *}$ & 54.73 & $0.43^{* * *}$ & 52.61 & $0.14 * * *$ & 28.27 \\
\hline 2016 & 65.89 & -196.07 & 28.08 & -3.59 & 2.47 & -14.55 & $1.82 * * *$ & 177.74 & $1.26^{* * *}$ & 31.17 & $0.33^{* * *}$ & 40.73 & $0.16^{* * *}$ & 15.19 \\
\hline 2017 & 69.15 & -314.15 & 25.53 & -5.73 & 1.88 & -21.65 & $1.95 * * *$ & 183.43 & $1.14 * * *$ & 44.08 & $0.24 * * *$ & 44.31 & $0.10^{* * *}$ & 21.79 \\
\hline All & 65.58 & -242.20 & 25.24 & -13.18 & 2.51 & -31.91 & $3.69 * * *$ & 206.80 & $1.97 * * *$ & 73.55 & $0.75^{* * *}$ & 65.00 & $0.25 * * *$ & 30.27 \\
\hline
\end{tabular}


Table 3.Composition of the closed motif

Table 3 lists the top five closed motif types with the highest frequency of occurrence. The node color represents the shareholder category. The red node represents FIC, the yellow node represents GC, the green node represents II, the black node represents SOC, and the blue node represents QFII.

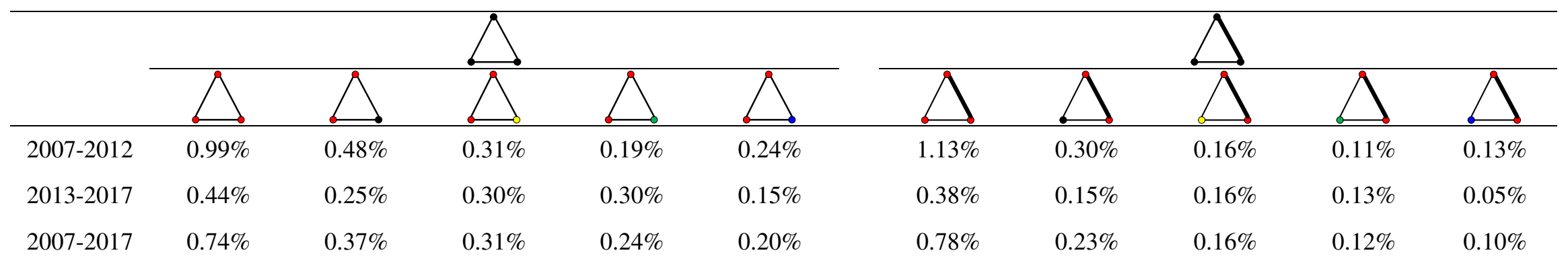

\begin{tabular}{|c|c|c|c|c|c|c|c|c|c|c|}
\hline $2007-2012$ & $0.62 \%$ & $0.07 \%$ & $0.07 \%$ & $0.04 \%$ & $0.02 \%$ & $0.22 \%$ & $0.03 \%$ & $0.02 \%$ & $0.02 \%$ & $0.00 \%$ \\
\hline $2013-2017$ & $0.16 \%$ & $0.04 \%$ & $0.04 \%$ & $0.00 \%$ & $0.04 \%$ & $0.07 \%$ & $0.03 \%$ & $0.01 \%$ & $0.01 \%$ & $0.01 \%$ \\
\hline $2007-2017$ & $0.41 \%$ & $0.06 \%$ & $0.05 \%$ & $0.02 \%$ & $0.02 \%$ & $0.15 \%$ & $0.03 \%$ & $0.01 \%$ & $0.01 \%$ & $0.00 \%$ \\
\hline
\end{tabular}




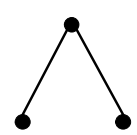

(1)

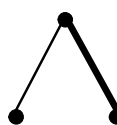

(2)



(3)

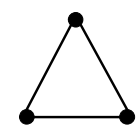

(4)

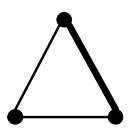

(5)

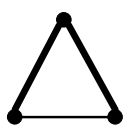

(6)



(7)

Figure 1. Seven weighted 3-node motifs

Figure 1 shows seven weighted 3-node motifs. The thick line is the strong co-holding relationship, and the thin line is the weak co-holding relationship.

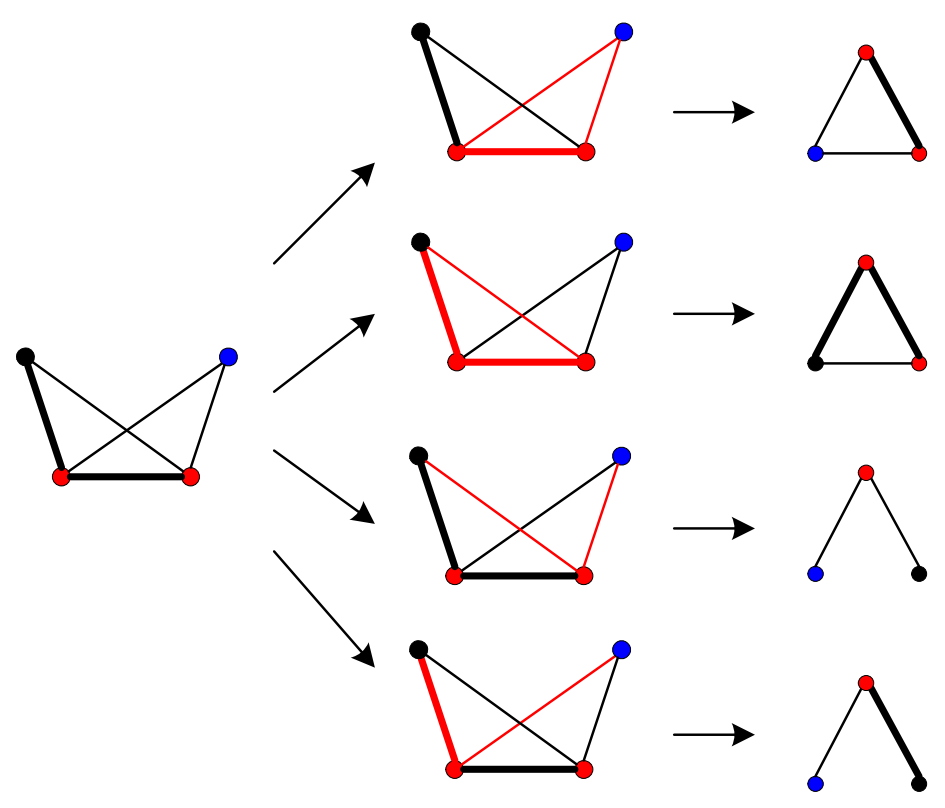

Figure 2. Schematic figure of motif identification

Figure 2 shows the schematic figure of motif identification. The node color represents the shareholder category, where the red node represents FIC, the black node represents SOC, and the blue node represents QFII. The red edges represent the process of motif identification. 


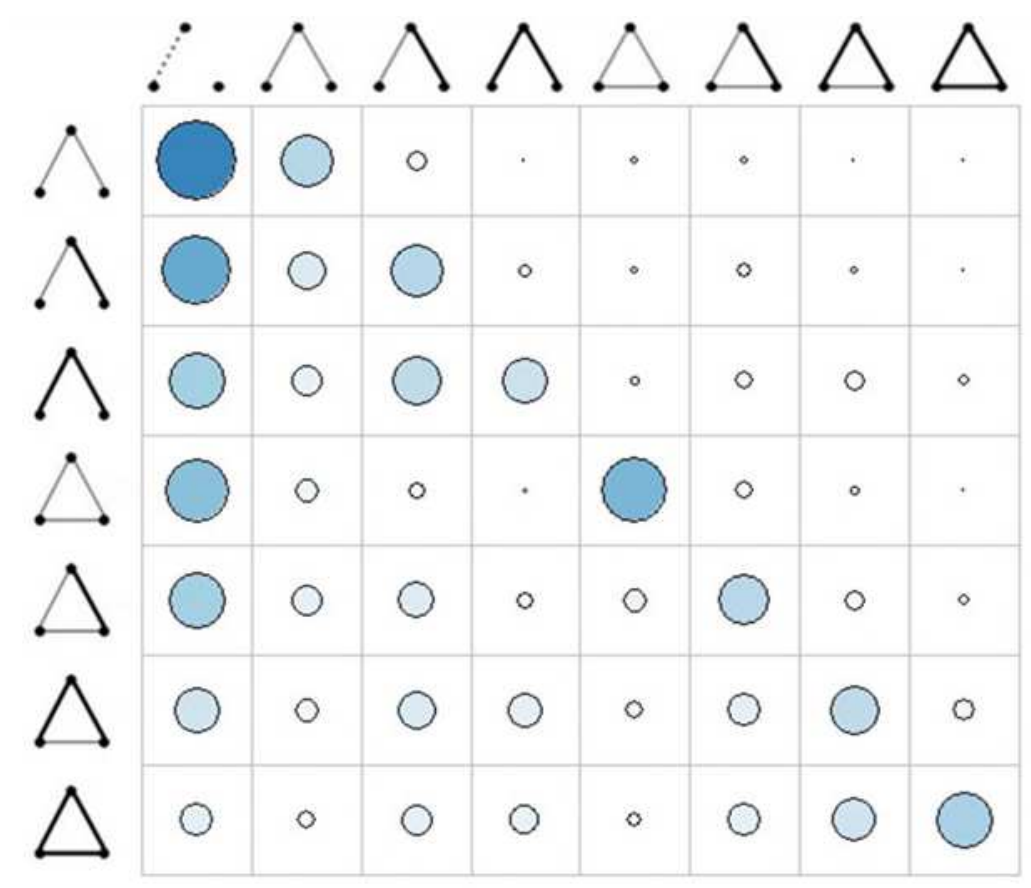

Figure 3. Motif evolution in the short term

Figure 3 shows the motif evolution in the short term. The vertical axis is the motif structure in the previous period, and the horizontal axis is the motif structure in the later period. The circle (and the shade of the color) represents the evolution probability, which is the average of all quarters. 


\begin{tabular}{|c|c|c|c|c|c|c|c|c|c|}
\hline \multirow[t]{2}{*}{ Motif } & \multicolumn{3}{|c|}{ Invariant motif } & \multicolumn{3}{|c|}{ Vanishing motif } & \multicolumn{3}{|c|}{ Transforming motif } \\
\hline & $1 \mathrm{st}$ & 2nd & $3 \mathrm{rd}$ & $1 \mathrm{st}$ & 2nd & $3 \mathrm{rd}$ & $1 \mathrm{st}$ & 2nd & $3 \mathrm{rd}$ \\
\hline & $(0.81)$ & $(0.76)$ & $(0.65)$ & $(0.74)$ & $(0.74)$ & $(0.73)$ & $(0.12)$ & $(0.10)$ & $(0.09)$ \\
\hline & $(0.67)$ & $(0.56)$ & $(0.56)$ & $(0.71)$ & $(0.71)$ & $(0.65)$ & $(0.32)$ & $(0.31)$ & $(0.30)$ \\
\hline & $(0.55)$ & $(0.54)$ & $(0.52)$ & $(0.47)$ & $(0.45)$ & $(0.40)$ & $(0.56)$ & $(0.53)$ & $(0.50)$ \\
\hline & $(0.98)$ & $(0.95)$ & $(0.94)$ & $(0.56)$ & $(0.55)$ & $(0.51)$ & $(0.27)$ & $(0.21)$ & $(0.14)$ \\
\hline & $(0.79)$ & $(0.78)$ & $(0.77)$ & $(0.40)$ & $(0.39)$ & $(0.36)$ & $(0.49)$ & $(0.46)$ & $(0.44)$ \\
\hline & $(0.96)$ & $(0.84)$ & $(0.72)$ & $(0.33)$ & $(0.27)$ & $(0.23)$ & $(0.58)$ & $(0.57)$ & $(0.57)$ \\
\hline & $(0.62)$ & $(0.60)$ & $(0.58)$ & $(0.12)$ & $(0.12)$ & $(0.08)$ & $(0.58)$ & $(0.52)$ & $(0.47)$ \\
\hline
\end{tabular}

Figure 4. Motif evolution in the short term considering node attributes 
Figure 4 shows motif evolution in the short term considering node attributes. 1st, 2nd, and 3rd respectively represent the evolution probability of the top 3 motifs. The number in parentheses indicates the probability of motif evolution. Taking 0.81 in the upper left corner of the figure as an example, it means that in the evolutionary motif where motif 1 remains invariant, the probability of the motif with all three nodes being GC is the highest, which is 0.81 . In other words, there are 100 motifs 1 with all three nodes as GC, and 81 motifs 1 remain unchanged in the next quarter. The node color represents the shareholder category. The red node represents FIC, the yellow node represents GC, the green node represents II, the black node represents SOC, and the blue node represents QFII. 


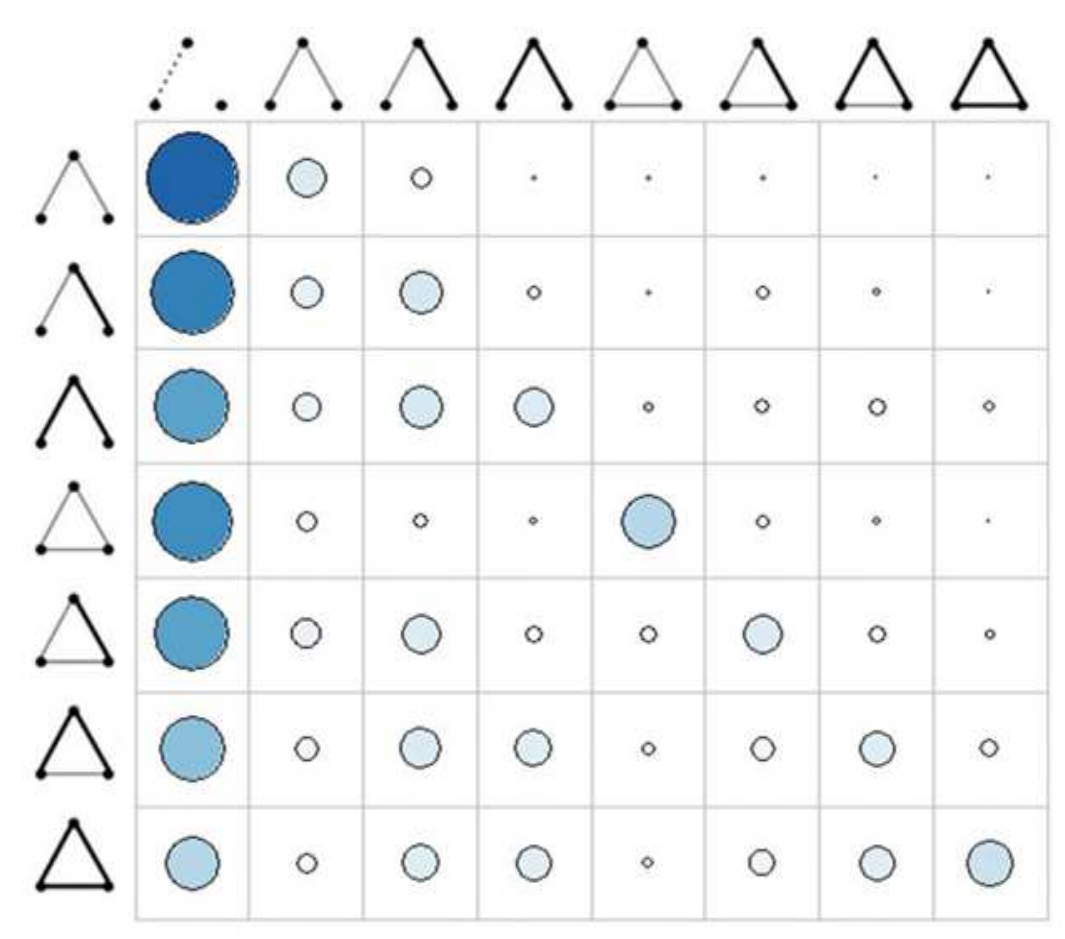

Figure 5. Motif evolution in the long term

Figure 5 shows the motif evolution in the long term. The vertical axis is the motif structure in the previous period, and the horizontal axis is the motif structure in the later period. The circle (and the shade of the color) represents the evolution probability, which is the average of all quarters. 


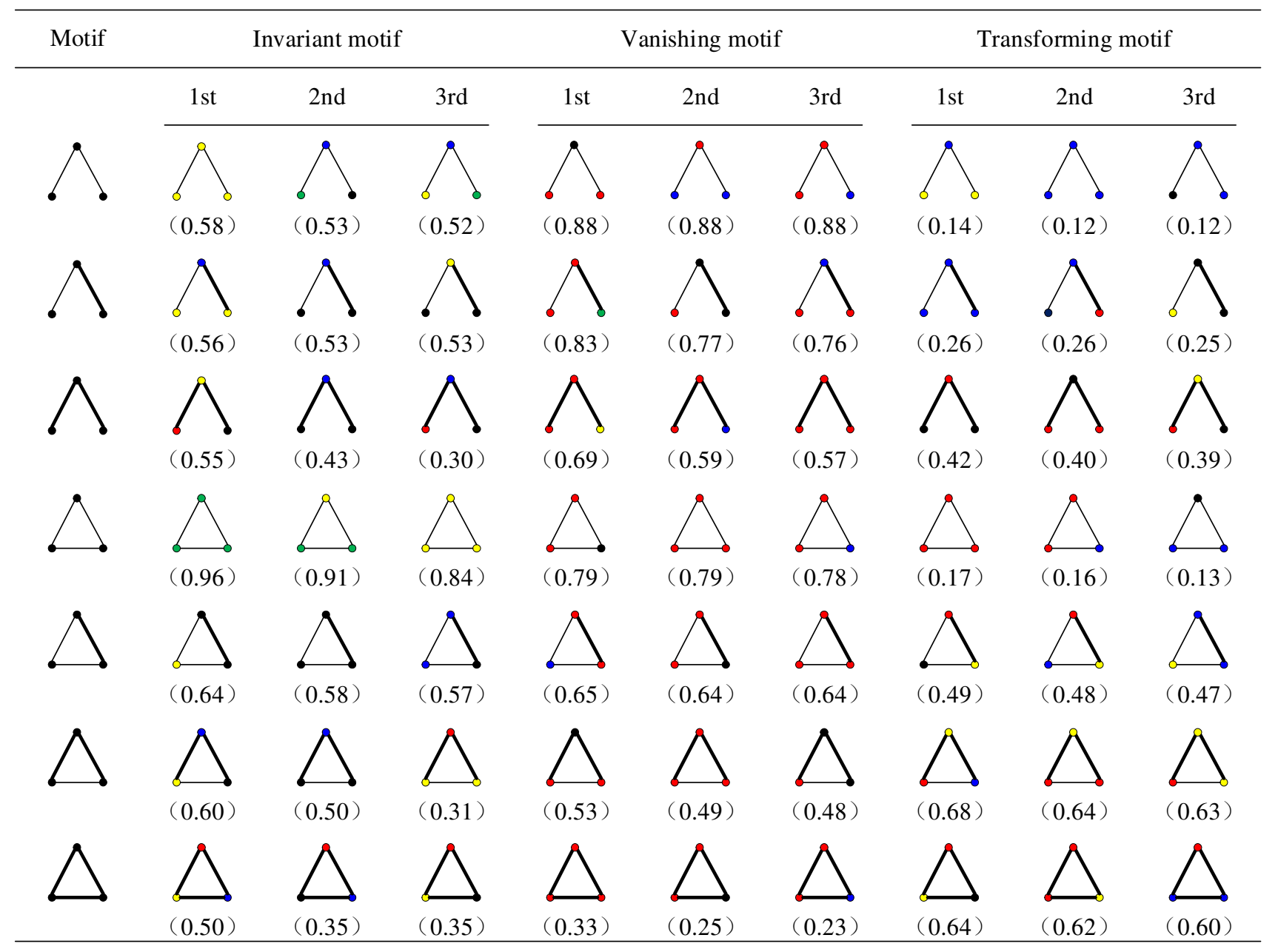

Figure 6. Motif evolution in the long term considering node attributes 
Figure 6 shows motif evolution in the long term considering node attributes. 1st, 2nd, and 3rd respectively represent the evolution probability of the top 3 motifs. The number in parentheses indicates the probability of motif evolution. The node color represents the shareholder category. The red node represents FIC, the yellow node represents GC, the green node represents II, the black node represents SOC, and the blue node represents QFII. 